\title{
ON THE MULTIPLICATIVE STRUCTURE OF THE DE RHAM THEORY
}

\author{
V. K. A. M. GUGENHEIM
}

Let $\mathscr{T}$ denote either the category of $C^{\infty}$-manifolds (possibly with boundaries and corners) and $C^{\infty}$-maps, or that of simplical complexes and simplicial maps. Let $\mathscr{V}$ denote the category of graded differential vector spaces over the reals or the rationals, and let $A^{*}: \mathscr{T} \rightarrow \mathscr{V}$ denote either the classical de Rham functor of differential forms, or Sullivan's functor of rational differential forms; cf. [4]. By $C^{*}: \mathscr{T} \rightarrow \mathscr{V}$ we denote, in the two cases, the functor of either normalized singular differentiable cochains or normalized simplicial cochains. The differentiable cochain (or chain) functor is homology equivalent to the usual (continuous) singular functors. This is well-known; for a proof see, for instance, [7, 191-200].

The transformation of functors

$$
\rho: A^{*} \rightarrow C^{*}
$$

is defined by

$$
\langle\rho \omega, c\rangle=\int_{c} \omega
$$

where $c$ is any singular (or simplicial) chain. The de Rham theorem says, in either case, that $\rho$ induces a homology isomorphism. Additionally, the theorem asserts that the isomorphism

$$
H(\rho): H\left(A^{*}\right) \rightarrow H\left(C^{*}\right)
$$

is a map of algebras. Of course, $\rho$ itself is not a map of algebras ; $A^{*}$ is commutative and $C^{*}$ is not.

Situations of this sort-maps which are not multiplicative but become so in homology - are encountered elsewhere, and it has been observed that often this phenomenon is associated with the existence of a whole family of "higher homotopies"; cf. e.g. [6]. The maps of this sort form a category denoted by $D A S H$ in [5], of which the category $D A$ of differential algebras is a subcategory. In this paper we shall prove the following

Received September 18, 1974. 
Theorem. $\rho$ can be extended to a map of DASH.

The theorem means that, writing $\rho=\rho_{1}$ we can find

$$
\rho_{i}: A^{*(i)} \rightarrow C^{*} \quad(i \geq 1)
$$

where $A^{*(i)}=A^{*} \otimes \cdots \otimes A^{*}$ ( $i$ times), and $\otimes$ denotes the tensor product over the reals or rationals as the case may be, such that

(i) $D \rho_{i}=\sum_{j=1}^{i-1}(-1)^{j}\left\{\phi\left(\rho_{j} \otimes \rho_{i-j}\right)-\rho_{i-1}\left(A^{(j-i)} \otimes \phi \otimes A^{(i-j-1)}\right)\right\}$,

(ii) $\rho_{i}\left(\omega_{1} \otimes \cdots \otimes \omega_{i}\right)=0$ if $i \geq 2$ and one of the $\omega$ 's is a constant 0 -form (on each component).

Here $D \rho_{i}=d \cdot \rho_{i}+(-1)^{i} \rho_{i} \cdot d$, where we note that $\rho_{i}$ has degree $-i+1$ and $\phi$ stands, generically, for multiplication. Thus

$$
\begin{aligned}
\phi(\alpha \otimes \beta) & =\alpha \wedge \beta \text { (exterior product) } & & \text { if } \alpha, \beta \in A^{*}(x) \\
& =\alpha \cup \beta \text { (cup product) } & & \text { if } \alpha, \beta \in C^{*}(x) .
\end{aligned}
$$

For $i=1,2$ we get

$$
D \rho_{1}=0, \quad D \rho_{2}=\rho_{1} \phi-\phi\left(\rho_{1} \otimes \rho_{1}\right) .
$$

The latter statement contains, of course, the classical result that $H(\rho)$ is multiplicative. The main application which the author has in mind is the following: Let $E \rightarrow X$ be a fibration, and $Y \rightarrow X$ a map. Then, by a theorem of Eilenberg and Moore [3] the cohomology of the induced space is given by the differential Tor functor

$$
\operatorname{Tor}_{C^{*}(X)}\left(C^{*} Y, C^{*} E\right) .
$$

Now, consider the diagram

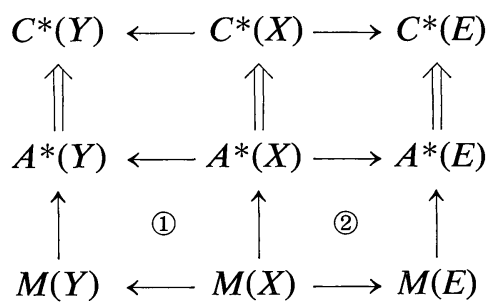

where $\Rightarrow$ denotes the map of $D A S H$ given by our theorem, and $M(X)$ the "minimal algebra" of Sullivan ; cf. [4]. Since $M(X)$ is not functorial, the diagrams (1) and (2) are not commutative; it is true, however, that they are homotopy-commutative in the sense of the category $D A S H$. Hence, by the principal theorem of [5],

$$
\operatorname{Tor}_{C^{*}(X)}\left(C^{*}, Y, C^{*}(E)\right) \leftarrow \operatorname{Tor}_{M(X)}(M(Y), M(E))
$$


exists and is an isomorphism. In other words, all the calculations can be made at the level of the minimal algebras. This provides a very direct approach to Sullivan's theory, the details of which will be discussed elsewhere.

The same remark is one way of justifying the use of the de Rham complex in the proof of Theorem 2.1 in [1] by P. F. Baum and L. Smith.

Since $\rho_{i}$ has degree $-i+1, \rho_{i} \mid\left(A^{(i)}\right)^{n}=0$ if $n<i-1$, where $\left(A^{(i)}\right)^{n}$ denotes the elements of dimension $n$. Thus, is dimension $0, \rho_{i}=0$ if $i>1$. This is possible since $\rho_{1}(\alpha \wedge \beta)=\rho_{1} \alpha \cup \rho_{1} \beta$ if $\alpha, \beta$ are 0 -forms, i.e., functions. We shall now prove the existence of the $\rho_{i}$ by induction assuming that $\rho_{j}$ has been determined for $j<i$, and finding $\rho_{i} \mid\left(A^{i}\right)^{n}$ by induction on $n$. As we noted, $\rho_{i} \mid\left(A^{i}\right)^{n}=0$ if $n<i-1$.

We shall use the machinery of (contravariant) acyclic models, which was introduced (covariantly) by Eilenberg and MacLane [2]. In its basic idea the proof is merely a technical elaboration of the argument given by Whitney for the uniqueness of the cup product, [8]; see also [9, p. 143].

For any contravariant functor $K: \mathscr{T} \rightarrow$ vector spaces we define a new contravariant functor $\hat{K}: \mathscr{T} \rightarrow$ vector spaces by

$$
\hat{K}(X)=\prod_{u: M \rightarrow X}\{K(M), u\}
$$

where the product is over all maps $u: M \rightarrow X$ of $\mathscr{T}$, and $M$ is a "model", i.e., one of the standard simplexes $\Delta^{n}$. If $f: X \rightarrow Y$ is a map, then $\hat{K}(f): \hat{K}(Y) \rightarrow$ $\hat{K}(X)$ is defined by

$$
\hat{K}(f)\left\{m_{v}, v\right\}=\left\{m_{f u}, u\right\},
$$

where $u: M \rightarrow X, v: M_{v} \rightarrow Y$, and $m_{v} \in K\left(M_{v}\right)$.

If $K, L: \mathscr{T} \rightarrow \mathscr{V}$ are contravariant functors, and $\theta: K \rightarrow L$ is a transformation of functors, then we define the tranformation of functors $\hat{\theta}: \hat{K} \rightarrow \hat{L}$ by

$$
\hat{\theta}(x)\{m, u\}=\{\theta(M) m, u\} .
$$

For any contravariant functor $K: \mathscr{T} \rightarrow \mathscr{V}$ we define $\Phi: K \rightarrow \hat{K}$ by

$$
\Phi(X) h=\{K(u) h, u\} \quad(h \in K(X)) .
$$

We easily verify that

$$
\hat{\theta} \Phi=\Phi \theta
$$

In particular, for the functors $A^{*}$ and $C^{*}$ we define $\hat{A}^{*}$ and $\hat{C}^{*}$ by

$$
\left(\hat{A}^{*}\right)^{n}=\left(\widehat{\left.A^{*}\right)^{n}}, \quad\left(C^{*}\right)^{n}=\left(\widehat{\left.C^{*}\right)^{n}},\right.\right.
$$

and we get differentials 


$$
\hat{d}: \hat{A}^{*} \rightarrow \hat{A}^{*}, \quad \hat{d}: \hat{C}^{*} \rightarrow \hat{C}^{*} .
$$

Lemma 1. The functor $C^{*}$ is corepresentable, i.e., there is a transformation of functors $\Psi: \hat{C}^{*} \rightarrow C^{*}$ such that $\Psi \Phi=$ the identity.

Note that we do NOT assert $\Psi d=d \Psi$.

Proof. We define $\Psi(X)=\hat{C}^{*}(X) \rightarrow C^{*}(X)$ by

$$
\left\langle\Psi(X)\left\{m_{u}, u\right\}, v\right\rangle=\left\langle m_{v}, 1_{M_{v}}\right\rangle,
$$

where $v: M_{v} \rightarrow X$, and $1_{M_{v}}$ denotes the identity map of $M_{v}$ regarded as a singular chain. The verification of $\Psi \Phi=$ identity is trivial.

Lemma 2. The functors $A^{(r)}(r \geq 1)$ are acyclic on models; thus for each model $M$ there is a chain homotopy

$$
S_{M}: A^{(r)}(M) \rightarrow A^{(r)}(M)
$$

such that

$$
D S_{M}=A^{(r)}-\varepsilon_{M},
$$

where $\varepsilon_{M}$ is the augmentation; $S_{M}$ and $\varepsilon_{M}$ are only defined after a contraction has been chosen for $M$.

The lemma is clear for $r=1$ and then, for a general $r$, follows from the well-known definition of the tensor product.

For each model $M$ we choose, once and for all, an $S_{M}$. Now we define

$$
S: \hat{A}^{(r)}(X) \rightarrow \hat{A}^{(r)}(X)
$$

by

$$
S\left\{\omega_{u}, u\right\}=\left\{S_{M} \omega_{u}, u\right\},
$$

where $u: M \rightarrow X$. Clearly,

$$
D S=\hat{A}^{(r)}-\varepsilon,
$$

where $\varepsilon\left\{\omega_{u}, u\right\}=\left\{\varepsilon_{M} \omega_{u}, u\right\}$. Notice that $S$ is a transformation of functors.

We now begin our main proof. We write the defining equations for $\rho_{i}$ as

$$
\begin{aligned}
\rho_{i} d & =(-1)^{i}\left\{-d \rho_{i}+\sum_{j=1}^{i-1}(-1)^{j} \phi\left(\rho_{j} \otimes \rho_{j-1}\right)-\rho_{i-1}\left(A^{j-i} \otimes \phi \otimes A^{i-j-1}\right)\right\} \\
& =\theta_{i} \quad \text { say. }
\end{aligned}
$$

We assume that $\rho_{j}$ is defined for $j<i$, and $\rho_{i}$ in dimension $<n$, where $n \geq i-1$, so that $n \geq 1$ since $i \geq 2$. Thus $\theta_{i}$ is defined in dimension $n-1$, and we have the following diagram: 


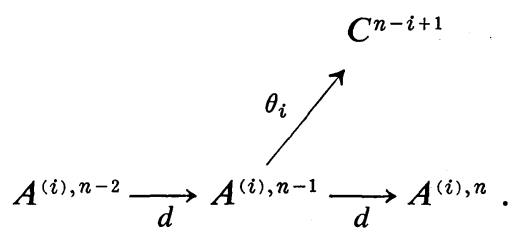

By the inductive hypothesis, $\theta_{i} d=0$.

Since everything is functorial, we get the diagram

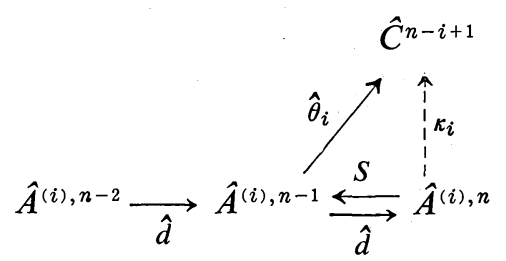

where $\hat{\theta}_{i} \hat{d}=0$. Now we define $\kappa_{i}=\hat{\theta}_{i} S$ and obtain

$$
\kappa_{i} \hat{d}=\hat{\theta}_{i} S \hat{d}=\hat{\theta}_{i}(1-\varepsilon-\hat{d} S)=\hat{\theta}_{i},
$$

where we have used $\theta_{i} \varepsilon=0$. This is clear for $i>2$ since $\theta_{i}$ has degree $-i+2$. We also have

$$
\theta_{2}=-d \rho_{2}+\phi\left(\rho_{1} \otimes \rho_{1}\right)-\rho_{1} \phi,
$$

which is zero in dimension 0 as was remarked before.

Now define $\rho_{i}$ on $A^{(i), n}$ by

$$
\rho_{i}=\Psi_{i} \Phi
$$

and verify

$$
\rho_{i} d=\Psi \kappa_{i} \Phi d=\Psi \kappa_{i} \hat{d} \Phi=\Psi \hat{\theta}_{i} \Phi=\Psi \Phi \theta_{i}=\theta_{i}
$$

as required.

It remains to check condition (ii) of the theorem. For the moment, say the element

$$
\omega=\omega_{1} \otimes \cdots \otimes \omega_{i} \in A^{(i)}(X), \quad i \geq 2,
$$

is "special" if at leasto ne $\omega_{j}$ is a constant 0 -form. Now notice that if $\omega$ is special and $\rho_{i}$ satisfies the condition inductively, then $\theta_{i} \omega=0$ when $\omega$ is special, as can be easily verified from the formula. Also $\Phi(X) \omega=\left\{u^{*} \omega, u\right\}$, and if $\omega$ is special so is $u^{*} \omega=A^{(i)}(u) \omega$. Hence $\Phi$ sends special forms to special forms. $S_{M}$ of a constant form is 0 , from which we deduce easily that $S_{m} \omega$ is special if $\omega$ is special. Thus inductively $\kappa_{i} \Phi=\rho_{i} S \Phi$ will carry special forms to zero, 
and the same is true of $\rho_{i}$. Hence the proof of our theorem is complete.

\section{References}

[1] P. F. Baum \& L. Smith, The real cohomology of differentiable fibre bundles, Comment. Math. Helv. 42 (1967) 171-179.

[2] S. Eilenberg \& S. MacLane, Acyclic models, Amer. J. Math 75 (1953) 189-199.

[ 3 ] S. Eilenberg \& J. C. Moore, Homology and fibrations. I, Comment. Math. Helv. 40 (1966) 199-236.

[4] E. Friedlander, P. A. Griffiths \& J. Morgan, Homotopy theory and differential forms, Mimeographed notes, Seminar of Geometry, Florence, 1972.

[5] V. K. A. M. Gugenheim \& H. Munkholm, On the extended functoriality of Tor and Cotor, J. Pure Appl. Algebra, 1974.

[6] J. Stasheff \& S. Halperin, Differential homological algebra in its own rite, Proc. Advanced Study Inst. Algebraic Topology, Aarhus, 1970.

[7] F. W. Warner, Foundations of differentiable manifolds and Lie groups, Scott \& Foresman, Glenview, Illinois, 1971.

[ 8 ] H. Whitney, On products in a complex, Ann. of Math. 39 (1938) 397-432.

[9] —, Geometric integration theory, Princeton University Press, Princeton, 1957.

University of Illinois, Chicago Circle 